\title{
La enseñanza de la historia en las aulas: un tema para reflexionar
}

\section{Teaching history in the classroom: a topic to reflect on

\author{
Deilyn Lahera Prieto* \\ Francisco Alberto Pérez Piñón**
}

\begin{abstract}
* Universidad Autónoma de Chihuahua, México. Es Licenciada en Ciencias de la Educación por la Universidad de Ciencias Pedagógicas (UCP)Frank País, Cuba. Actualmente cursa el programa de Maestría en Innovación Educativa, que se encuentra incorporado en el Padrón Nacional de Posgrados de Calidad (PNPC) del Consejo Nacional de Ciencia y Tecnología (CONACyT). Correo electrónico: deilynlahera1@gmail.com

(1) https://orcid.org/0000-0002-3576-3230
\end{abstract}

** Profesor Investigador de la Universidad Autónoma de Chihuahua, México. Es doctor en Ciencias Pedagógicas. Entre sus publicaciones recientes están "Fundamentos teórico-metodológicos en la investigación educativa en Chihuahua; análisis de un área del conocimiento" en IE Revista de Investigación Educativa de la REDIECH (2019) y el capítulo de libro "Conciencia histórica en la oralidad y lo documental" en La historia oral: usos y posibilidades en la investigación histórico-educativa (2021). Cuenta con reconocimientos Prodep y del Sistema Nacional de Investigadores, Nivel 1. Es miembro de la Sociedad Mexicana de Historia de la Educación, del Consejo Mexicano de Investigación Educativa y de la Red de Investigadores Educativos Chihuahua. Correo electrónico: aperezp@uach.mx

(1) https://orcid.org/0000-0003-4316-6484

Historial editorial

Recibido: 22-marzo-2020

Aceptado: 03-diciembre-2020

Publicado: 29-enero-2021

ISSN-e: 2594-2956 
La enseñanza de la historia en las aulas: un tema para reflexionar

\section{Resumen}

La significación que amerita la enseñanza de la historia en niveles de educación media, está siendo en la actualidad objeto de estudio para los investigadores de esta ciencia. En los últimos quince años la investigación educativa relacionada con la didáctica de la historia, ha sido testigo de un fuerte avance, sin embargo, una parte importante de estudiantes demuestra desinterés por esta área del conocimiento (materia), necesitándose la implementación de nuevas estrategias metodológicas, más motivadoras y más acordes a los tiempos en que vivimos. Se requiere entonces de la disposición del estudiante para instruirse y de la intervención del docente en esa dirección. Mejorar la didáctica en la enseñanza de la historia y los métodos empleados en el aula, es hoy un reto, por lo que se requiere cambiar la mirada y realizar propuestas que promuevan la vinculación del contenido teórico con la praxis. La formación histórica correcta de los estudiantes demanda un cambio, por lo que es menester del aprendizaje histórico actual, la asunción de nuevos retos que colaboren con el desarrollo cognitivo de los estudiantes en cualesquiera de los niveles de enseñanza, posibilitando el interés de estos por la materia, su involucramiento intelectual y por ende, el aprendizaje de esta materia más allá de fechas, hechos y acontecimientos. No se omite enunciar que el presente ensayo es parte de un trabajo de investigación más amplio, lo que aquí se presenta es lo

130 concerniente al enfoque teórico asumido como sustento de la investigación.

Palabras clave: enseñanza de la historia, cultura histórica, conciencia histórica, memoria histórica.
Teaching history in the classroom: a topic to reflect on

\section{Abstract}

The significance of teaching history in secondary educational levels is currently being studied by researchers in this science. During the last decade, educational research in teaching history has witnessed overwhelming progress; however, students continue to show disinterest in this subject, requiring thus the implementation of new methodological strategies, which ought to be motivating, taking into consideration the challenges and the impediments faced in this area. Therefore, both the willingness of the learners to educate themselves and the intervention of the teachers are required. There is a need for improving the didactic approach and methodology in the classroom for the teaching of history to be able to make a proposal that promotes the linking of the theoretical contents to practical elements. The appropriate historical training of students demands a change; hence, it is necessary for the current teaching and learning of history the assumption of new challenges that promote the cognitive development of students at any educational levels, encouraging their interest in this subject, their intellectual involvement and therefore, creating a new ideology of learning beyond the previous focus on dates, facts, and events. The present paper is part of larger-scale research work; what is presented here focuses on the theoretical approach assumed as the basis of the research.

Keywords: teaching history, historical culture, historical consciousness, historical memory. 
L'enseignement de l'histoire dans les salles de classe : un sujet de réflexion

\section{Résumé}

L'importance de l'enseignement de l'histoire au niveau de l'enseignement secondaire est actuellement à l'étude pour les chercheurs de cette science. Au cours des quinze dernières années, la recherche éducative liée à la didactique de l'histoire a été témoin d'une percée, mais une partie importante des étudiants montre un désintérêt pour ce domaine de la connaissance (matière)de nouvelles stratégies méthodologiques, plus motivantes et mieux adaptées à notre époque. Il faut alors que l'étudiant soit disposé à s'instruire et que l'enseignant intervienne dans cette direction. L'amélioration de l'enseignement didactique dans l'enseignement de l'histoire et des méthodes employées dans la salle de classe est aujourd'hui un défi, et il est donc nécessaire de changer le regard et de faire des propositions qui favorisent le lien entre le contenu théorique et la pratique. La formation historique correcte des étudiants exige un changement, ce qui nécessite l'apprentissage historique actuel, la prise en charge de nouveaux défis qui contribuent au développement cognitif des élèves à tous les niveaux d'enseignement, en permettant à ceux-ci de s'intéresser à la matière, à son implication intellectuelle et donc à l'apprentissage de cette matière au-delà des dates, des faits et des événements. Il n'est pas omis d'indiquer que cet essai fait partie d'un travail de recherche plus large, ce qui est présenté ici, c'est l'approche théorique adoptée comme base de la recherche.

Mots-clés: enseignement de l'histoire, culture historique, conscience historique, mémoire historique.
Nauczanie historii w szkole sredniej: temat do refleksji

\section{Streszczenie}

Nauczanie historii na poziomie szkoly sredniej jest obecnie szeroko dyskutowanie przez badaczy zajmujacych sie dydaktyka historii. W ciągu ostatnich piętnastu lat badania edukacyjne związane $\mathrm{z}$ dydaktyką historii były świadkami postępu, jednak duza część uczniów wykazuje brak zainteresowania tym obszarem wiedzy (materii), wymagającym wdrożenia nowych strategii metodologicznych, bardziej motywującymi i bardziej zgodnymi z czasami, w którym żyjemy. Wymagana jest wówczas gotowość ucznia do samodoksztalcania i interwencja nauczyciela w tym kierunku. Poprawa nauczania historii i metod stosowanych w szkole jest wyzwaniem, dlatego konieczna jest zmiana strategii i zaproponowanie ideii powiązania treści teoretycznych z praktyką. Prawidłowe szkolenie w zakresie historii wymaga zmiany, więc konieczne jest obecne założenie nowych wyzwań, które współpracują z rozwojem poznawczym uczniow na każdym poziomie nauczania, umożliwiając im zainteresowanie tym przedmiotem. Ich zaangażowanie intelektualne polegajace na uczeniu się tego przedmiotu nie tylko na podstawie dat , faktow i wydarzen. Esej jest częścią szerszej pracy badawczej zajmujacej sie dydaktyka historii.

Slowa kluczowe: nauczanie historii, kultura historyczna, świadomośc historyczna, pamięc historyczna. 
"Se requiere generar un entorno donde el alumno
aprenda haciendo, experimentando y viviendo los conceptos"

David Fischman

\section{Introducción}

En el acontecer histórico del proceso enseñanza-aprendizaje, las exigencias y demandas sociales han sido diversas, especialmente las referidas a la posibilidad de aplicación práctica de los contenidos históricos por parte del alumnado. Para Schmidt (2017), en la práctica docente, la centralidad del aprendizaje se ve como una problemática que enfrentan los educadores, específicamente los versados en la Didáctica de la Historia, por lo que defiende la idea de que el que sabe cómo aprender, sabrá cómo enseñar.

A decir de Téllez (2020) este proceso está siendo dominado por la instrucción, relegando a un segundo plano, lo educativo y precisamente la Historia, a nuestro juicio, está siendo sometida a este mero proceso de instruir, cuando en realidad debería contribuir a desarrollar aprendizajes mediante la adquisición de herramientas conceptuales, destrezas y procedimientos que favorezcan la capacidad de aplicarlos a la propia realidad del alumno, para enfrentarse críticamente al mundo.

El profesor debe ser capaz de dirigir adecuadamente el proceso de construcción de conocimientos de los estudiantes y estos últimos deben ser capaces de obtener el aprendizaje necesario para cada etapa lectiva. Es así, que tanto el docente como el estudiante, participan activamente en este proceso formativo. Esta noción supone -ante todo- cambiar la mirada y asumir la enseñanza de la historia en términos de desarrollo de la percepción, la interpretación y la orientación históricas (González, 2006, p. 2I).

Es menester del aprendizaje histórico actual, la asunción de nuevos retos que colaboren con el desarrollo cognitivo de los estudiantes en cualquiera de los niveles de enseñanza, posibilitando el interés de estos por la materia, su involucramiento intelectual y por ende, el aprendizaje de la historia más allá de fechas, hechos y acontecimientos. En este sentido (Fronza, 20I6) destaca varias dimensiones de la matriz 
de la Didáctica de la Historia que podrían ser vistas como desafíos, entre los que se encuentran, la dimensión de la interpretación (es decir, el sentido que para el alumnado debe tener lo que aprende); la dimensión de la orientación (en este caso, posibilitar la ubicación del estudiante en el tiempo, es decir, mostrar desde la práctica, la interpretación del tiempo en lo externo y en lo interno) y la dimensión relacionada con la motivación, donde -para Rüsen- el aprendiz debería aprender a ubicar sus objetivos de vida personales y colectivos, en el flujo del tiempo.

Diversas son las experiencias mostradas acerca de la didáctica de la clase y partiendo de los resultados obtenidos, se hace un llamado a la variedad de perspectivas a la hora de impartir la materia, de forma tal que el estudiantado se involucre, debata, opine, exponga sus puntos de vista e intérprete. La denominada Clase Histórica de Schmidt (2016), propone una estructura metodológica para la enseñanza de la historia diferente, donde se interrelacionan factores que se van dando de manera independiente durante la clase.

No son pocos los que coinciden en plantear que para enseñar historia es suficiente la transmisión de fechas y sucesos acaecidos en el pasado, con algunas valoraciones subjetivizadas y su memorización, sin percibir que esto permite que los estudiantes le resten la significación que, para su vida, puede generar uno de estos sucesos. En este sentido, Burenheide (2007), Zhao y Hoge (2005), refieren que esto contribuye a fomentar la confusión ya existente entre el pasado y la historia, por lo que se hace necesario enseñar en el aula a pensar históricamente, es decir, auxiliarse de una enseñanza explicita (Wineburg, 200I).

Son muchas las razones que inducen a pensar en una reestructuración en la forma de trabajar la enseñanza de la historia. Para Cuesta (I998), la enseñanza de la historia difiere del resto de las asignaturas y se aleja de la ciencia histórica, mientras que para Matozzi (1999), el enlace entre ellas depende de procesos de transposición didáctica. Para lograr más argumentos relacionados con el tema, se plantean en el escrito, las siguientes interrogantes: ¿Muestran interés los estudiantes por el aprendizaje de la Historia? ¿Utilizan los docentes la metodología adecuada para impartir la asignatura de historia? 


\section{Situación actual de la enseñanza de la Historia}

Generalmente, cuando se analizan los diferentes problemas que se dan con la enseñanza de la historia, se observa que guardan estrecha relación con sus finalidades y con los usos sociales que las personas le dan a este importante saber escolar. De igual manera, se encuentran dificultades con los contenidos que se seleccionan para ser enseñados en la escuela y con los métodos de enseñanza y aprendizaje utilizados. Jesús Marolla y Joan Pagés (2018) en estudios realizados en Argentina, Chile y Colombia, ponen de relieve las limitaciones de la enseñanza de hechos y procesos históricos recientes y las repercusiones que ello puede tener para la comprensión histórica del presente, por parte del alumnado. Demuestran que el conocimiento didáctico de la historia que se enseña y se aprende ha de servir para aproximarse creativamente al pasado, relacionarlo con el presente y así poder entender su historicidad para proyectarse al futuro.

Para Herrera (2018), en varios niveles escolares los estudiantes han manifestado que la asignatura de historia tiende a ser una materia aburrida, ya que solamente para ellos es memorizar gran parte del contenido que el maestro les brinda durante todo un turno de clases, para luego realizar tareas extra clases. De igual manera, los autores Benítez, Gonda, y Pereira (2018), en investigaciones realizadas en algunos países como Estados Unidos y España, pudieron apreciar que los estudiantes de bachiller tenían como prioridad otras asignaturas que ellos declaraban como más importantes para ellos en su programa educativo, como lo eran las ciencias exactas o las de ciencias naturales. Agregaban que la historia era más fácil para estudiarla para aprobar un examen, por lo que le daban más importancia -al momento de estudiara otras asignaturas que para ellos eran llamadas como las más complejas.

Si pretendemos mejorar o incentivar el interés de los estudiantes por la asignatura, es urgente renovar los métodos que se están empleando en los centros escolares para impartir. La situación actual de la enseñanza de la historia se vuelve preocupante, es necesario realizar un estudio investigativo y realizar estrategias innovadoras que contribuyan al 
desarrollo del pensamiento crítico de los estudiantes y a su vez enriquecer las categorías propias de la historia como es la narración.

Es así que continúa siendo de interés reformar la manera en que se trabaja la enseñanza de la historia, para lo cual se requiere tomar en cuenta el criterio de los estudiantes, de manera que se puedan cubrir las necesidades que demandan y hacer más fructífero el proceso. Es el momento de involucrar más a los alumnos y desarrollar en ellos habilidades de segundo orden, donde el aprendizaje de esta materia sea algo más que la memorización de importantes fechas, hechos históricos o personajes de ciertos periodos.

En el ámbito universitario es habitual hablar acerca de innovación educativa y nuevas metodologías asociadas a paradigmas basados en el aprendizaje autónomo, por ejemplo: el trabajo cooperativo o la tutoría proactiva. Sin embargo, sigue existiendo una importante brecha entre la teoría y la práctica docente; una grieta que es aún mayor en el ámbito de las ciencias sociales, y más concretamente en la enseñanzaaprendizaje de la historia. Vivimos en una sociedad cada vez más centrada en la importancia de los conocimientos de carácter científico o técnico, en la que materias como matemática, física, química o ciencias naturales, tienen mayor relevancia en el currículo de educación de distintos niveles educativos.

La situación radica en que las ciencias sociales, a pesar de tratarse de ciencias, a menudo no son consideradas como tal, sino que son vistas como materias puramente culturales, directamente relacionadas con el discurso oral y aprendizaje memorístico, y -por lo tanto- no dan lugar al desarrollo de actividades de carácter discursivo, de indagación o de resolución de problemas (Prats y Santacana, 1998). Esta concepción de las ciencias sociales, hace patente la necesidad de dar un nuevo impulso a la pedagogía y didáctica de las mismas. Si queremos que el alumnado tenga interés por estas materias debemos dar un paso hacia delante e intentar implementar nuevas estrategias metodológicas más motivadoras y más acordes a los tiempos en que vivimos. 
Nos encontramos completamente inmersos en la "sociedad de la imagen". Hacemos uso sistemático de la tecnología en nuestro día a día y sin importar dónde nos encontremos, los estímulos visuales nos rodean por todas partes, los cuales debemos aprovecharlos desde las ciencias sociales en bien de una didáctica y pedagogía para estas áreas de conocimiento. Contamos con un número casi infinito de imágenes a nuestra disposición, tanto en Internet (accesibles desde cualquier dispositivo móvil, tableta $\mathrm{u}$ ordenador) como en publicaciones impresas en papel, que nos ofrecen imágenes de gran calidad, relacionadas casi con cualquier tema; en donde, para el logro del interés por la historia, podemos imaginarla como la piedra angular de las motivaciones sustantivas.

Son muchos los estudios que se han realizado referidos a la enseñanza de esta asignatura. Aceituno y Collao (2018), expresan que existen muchas diferencias en cuanto al conocimiento histórico que es impartido en las escuelas y la manera en que los estudiantes les dan aceptación. En Estados Unidos, Vansledright (1997), citado por Herrera (20I8), argumenta que los alumnos no le atribuyen ningún valor significativo a la historia, la ven y la analizan como un conjunto de fechas, datos y acontecimientos que se tienen que aprender; de esta misma forma, en España, los alumnos confesaron que la asignatura de historia está basada en una serie de datos y valoraciones que se convierte en una verdad acabada.

Determinados alumnos afirman que la Historia, es una herramienta para entender el presente a la luz del pasado. En ésta [sic] categoría, los alumnos, analizan los sucesos del presente, relacionando su origen con hechos del pasado. Los alumnos analizan determinados acontecimientos históricos y los conectan con la problemática del mundo contemporáneo (Benítez, Gonda y Pereira 20I8, p. I8).

Sáiz y López (2015) muestran en sus estudios que los estudiantes emplean conceptos históricos muy pobres, al igual que realizan relatos históricos poco complejos, siendo esto el resultado de una enseñanza que solo promueve la memorización de contenidos, demostrando cierta incapacidad para construir memorias históricas que le sean favorables al aprendizaje. El aprendizaje memorístico es uno de los procesos que 
en la actualidad continúa siendo cuestionado y se siguen aportado alternativas al respecto, desde campos y países diferentes, con miras a su extinción.

En este sentido, autores como Bertram, Wagner y Trautwein (20I7) manifiestan que la enseñanza de los profesores de historia debe pasar más allá de la memorización de sus contenidos, que enseñen a los estudiantes a pensar históricamente, que sean capaces de plantearse problemas para así desarrollar memorias constructivas en ellos; que mediante las clases de historia sean capaces de formularse preguntas para una mejor comprensión e interpretación del pasado.

En investigaciones como las ya referidas han demostrado la necesidad de cambiar los métodos, técnicas y recursos utilizados por los docentes en el aula, pues la metodología didáctica que se utiliza para impartir las clases de historia se está convirtiendo en un punto clave para poder entender la educación que están recibiendo los estudiantes y la forma como ellos perciben dicha materia.

Estudios realizados en bachillerato (Lamoneda, 1998), esbozan que los profesores deben cuestionarse la influencia que tiene el pensamiento historiográfico en el proceso enseñanza-aprendizaje, así como los caminos que utilizan para que el alumno adquiera o construya el conocimiento; pues son adolescentes y se debe seleccionar adecuadamente los recursos didácticos a utilizar, que favorezcan el aprendizaje a partir de actitudes reflexivas.

Unos de los recursos actuales, usados por los profesores como herramientas, resultan ser las redes sociales, especialmente para la descarga de imágenes que emplean en sus presentaciones de Microsoft Power Point o similares. Sin embargo, el empleo de estas imágenes sólo se hace como elemento ilustrativo u ornamental, bien para acompañar al texto o bien como imagen decorativa que permanece proyectada de fondo, mientras el/la docente explica la materia, sin hacer referencia alguna ellas, por lo que no son identificadas como fuentes para extraer información, ni como elemento de apoyo para comprender los contenidos. 
Ante esta situación nos urge superar esa concepción simplista que, de manera generalizada, se tiene del uso de la imagen y darle el valor que realmente le corresponde, viéndola y entendiéndola como una producción de saber. Un ejemplo fehaciente de esto, se evidencia en la propuesta Aprender y enseñar historia a través de la imagen artística: una propuesta didáctica sobre la Revolución Francesa (Pérez, 20I6), en la que se emplea la imagen como principal recurso didáctico que ayuda a entender, interpretar y contextualizar el pasado. Pero va un paso más allá y se centra en el uso de las imágenes de índole artística, con la intención reivindicar el lugar, los beneficios y el valor didáctico del arte en la enseñanza.

Si nos detenemos un momento y analizamos la presencia del arte en el currículo de la Secundaria Básica Obligatoria no es difícil darse cuenta de que "no es regular, y su estudio no es significativo ni sistemático ya que queda diluido en temarios sobrecargados de otros contenidos" (Lozano, 20I3, p. 4). Por estas razones se impone ir eliminando de los currículos actuales la visión de que el aprendizaje de la historia es una simple memorización de contenidos e informaciones de narrativas académicas. Sin embargo, hay que apostar por el desarrollo de competencias que colaboren con la formación de personas capacitadas para afrontar los problemas sociales y ambientales desde esta perspectiva.

\section{Metodologías empleadas por los docentes de bachillerato para impartir la asignatura}

Aprender Historia no se reduce a memorizar hechos, fechas, acontecimientos y demás, como se ha venido ya enunciando, pues la

138 adquisición de conocimientos históricos requiere comprensión y se relaciona con complejos procesos de pensamiento. Por lo tanto, la ganancia de este tipo de pensamiento se logra mediante la participación gradual en la práctica del historiador. Para ello, se hace necesario que los profesores de Historia posean una teoría sólida sobre el pensamiento histórico y su comprensión, el aprendizaje de la disciplina 
y el progreso o perfeccionamiento del campo cognoscitivo de sus alumnos.

Para Monfort, Pagès y Santisteban (20II), pensar históricamente significa desarrollar una conciencia de la temporalidad, que entienda el pasado como herramienta para conocer el presente. Para ello, son necesarias capacidades relacionadas con la imaginación y la empatía, destrezas para la representación y habilidades para la interpretación de fuentes. Ellos y muchos autores más, han abordado todo lo relacionado con la necesidad de definir qué se debe enseñar en la historia y el valor educativo que se le debe dar a los conocimientos; algunos de ellos los enunciamos aquí: (Wineburg, 200I; Carretero y López, 2009; Gómez, Ortuño y Molina, 20I4; Prats y Santacana, 20II; Stearns, Seixas y Wineburg, 2000).

VanSledright (2014) ha establecido una división entre los contenidos históricos que se imparten en las aulas, definiendo los de primer orden (hechos, fechas, conceptos, etc.) y los de segundo orden, que son los de carácter estratégico, que posibilitan organizar los de primer orden, explicarlos y relacionarlos con la práctica del historiador y el desarrollo de las habilidades para pensar históricamente.

Los contenidos de la asignatura de historia permiten comprender y conocer los problemas sociales que se gestan con el paso del tiempo, lo que facilita al ser humano ubicarse en la vida actual. De igual manera, conocer el pasado les permitirá comprender con más posibilidades su paso por el presente y con visión hacia el futuro. Para lograr una didáctica más amena, sin abandonar la explicación magistral, algunos autores proponen una clase activa, donde los estudiantes sean los protagonistas, que se realicen en forma de debates dirigidos por los profesores, profundizando en los contenidos históricos programados, utilizando noticias de actualidad, recopiladas de los diferentes medios de comunicación, así como la utilización de relatos anecdóticos de personajes populares, que animen las clases y las hagan más interesantes. 
Duarte (2018) y Fuster (2015) plantean la necesidad de que se perfeccione el campo cognoscitivo de los alumnos sobre la historia, para lograr la utilización de diferentes vías que posibiliten que las clases sean más emotivas y no queden en una simple charla, donde se hable de acontecimientos y fechas que tienden a aburrir y a desinteresar a los estudiantes. Mediante la resignificación del pasado, utilizando la narración como herramienta cultural, pueden lograrse mejores resultados de acuerdo a lo establecido por Carretero y López, (2009) motivando el desarrollo de habilidades complejas de pensamiento, que también pueden ser facilitadas por la resolución de problemas históricos y los contextos socioculturales.

Otros recursos mnemotécnicos que pueden facilitan la asimilación de los contenidos históricos en los estudiantes, están relacionados con las visitas a los museos y sitios patrimoniales, la participación en eventos culturales, lectura de leyendas, utilización de la música, etc. Además, el profesor debe inculcar en los alumnos el estudio independiente, con el cual puedan motivarse e impulsar el desarrollo de habilidades que propicien el análisis, la síntesis, la valoración y la interpretación, con relación a los temas tratados en clases.

Son disímiles los problemas a los que se enfrentan los profesores en su práctica profesional: la falta de interés de los alumnos por la asignatura, la carencia de estrategias para motivarlos y lograr que no se aferren a la simple memorización del contenido y lograr realizar análisis que los ayuden a entender por si mismos los diferentes conceptos.

Para Montanares y Llancavil (20I6), Castellanos, Estupiñán y Cuesta (20I7), no basta con que el profesor tenga dominio pleno del contenido a impartir, sino que necesita crear mecanismos que conecten la enseñanza con los problemas de la actualidad, brindado de esta forma un apoyo social a los estudiantes. Una alternativa sería que se logren realizar debates que surjan de opiniones o criterios de los mismos alumnos en las clases, fomentando siempre el respeto.

La asignatura de historia es una de las materias más complejas al momento de su enseñanza, en muchos países, abusan políticamente de 
ella, haciendo de los ciudadanos personas adictas a su ideología, es por eso que proponerse desarrollar en los estudiantes el pensamiento crítico, es otro de los desafíos; construir en ellos una conciencia histórica que les ofrezca la oportunidad de comprender la sociedad por la que van transitando con el paso del tiempo y a su vez lo que acontece en el mundo actual.

De igual manera Gómez, Rodríguez y Mirete, (2016) exponen que los maestros que imparten la asignatura ponen en evidencia que los primeros contenidos que se ofrecen en edades tempranas, muestran una gran carga conceptual, lo que obliga a los estudiantes a memorizar gran parte de este. Además, eluden la importancia de darle más participación al estudiante, que ellos tomen más protagonismo en el proceso de aprendizaje, exponiendo sus criterios.

Muchos docentes reconocen en la Historia no sólo la posibilidad de aprender, sino reflexionar y ampliar opciones de pensamiento en el sujeto y la sociedad. La historia supone un tipo de inteligencia diferente del conocimiento sociológico, económico o político, por lo que uno de sus propósitos fundamentales es coadyuvar a que el hombre desarrolle la comprensión de su devenir histórico y el de la humanidad; por lo que queda claro que no se trata de delimitar fechas y narrar acontecimientos, sino de comprender por qué se dieron los hechos y de qué manera afectaron el desarrollo de los procesos sociales, económicos, políticos y culturales en determinada época y lugar; así como poder interpretar a su vez, cómo afectaron el presente y cómo pueden afectar el futuro de la región en la cual conviven.

Es necesario replantearse las formas, modelos y metodologías que se están empleando hoy en día en las aulas, para el aprendizaje de la historia. Todo se concentra en la memorización de sucesos importantes, el alumno no toma el papel de líder para exponer sus conocimientos. Las clases son tan monótonas que no pasan más allá del discurso del letrado, el estudiante no es capaz de poner en práctica las narrativas que le permitan enriquecer su pensamiento y la conciencia histórica. Las clases deben pasar del modelo tradicional donde el profesor orienta 
actividades para llevar a cabo el proceso, que no le permite al estudiante desarrollar conceptos y habilidades de mayor complejidad.

\section{Percepción de los estudiantes acerca de la asignatura de Historia}

Estudios recientes realizados en España han evidenciado que los resultados obtenidos en los exámenes de historia, para el ingreso a las universidades, reflejan la hegemonía de la memorización de contenidos actuales y conceptuales. Sin embargo, resulta destacable que habilidades necesarias como el análisis, el razonamiento o la interpretación de documentos, fundamentales para el aprendizaje del pensamiento histórico, prácticamente no se observan. De igual manera, son casi imperceptibles procesos lógicos como la argumentación histórica del estudiante y la representación narrativa del pasado, elementos que resultan claves para el desarrollo del pensamiento histórico (Sáiz y Fuster, 20I4).

Prats y Santacana (20II) resumen que la historia cumple una función formativa esencial, pues promueve el ejercicio de análisis de problemas de diferentes sociedades en diferentes tiempos. Por otra parte, permite comprender la complejidad de cualquier acontecimiento, de cualquier fenómeno actual y de cualquier proceso del pasado, utilizando el análisis de causas y consecuencias.

El desinterés de los estudiantes de secundaria básica y bachillerato por el aprendizaje de la historia, es reflejado por autores como Sanz, Molero, y Rodríguez (20I7), Ortega (20I5) y Sáiz y López (20I5), pues para ellos no pasa de ser una materia aburrida, en donde los docentes les dan

142 un discurso sobre acontecimientos históricos y datos que deben memorizar días antes de los exámenes. A su percepción es solo reproducir lo que se les trasmitió en las clases y consideran que la asignatura que reciben en su currículo estudiantil no necesita ser comprendida, con sólo memorizar las fechas y acontecimientos más importantes es suficiente para demostrar conocimientos de la materia. 
Para ellos tener conocimientos históricos significa ser poseedor de una gran memoria.

Gómez, Miralles y Molina (2015) ha expuesto que los estudiantes manifiestan que la historia no guarda ninguna relación con su presente, dejan claro que se resume en ella un gran conjunto de hechos que sucedieron hace muchos años y que no se vinculan con la sociedad en que se desarrollan cada día. En muchos de procesos que ocurren en el aula se puede apreciar cómo el maestro se convierte en el único protagonista y los estudiantes muestran muy poca participación, llegando a calificar las clases como monótona.

En estudios realizados por Prats (2017) se detalla lo que para los estudiantes representa la asignatura, pues muchos coinciden en plantear que no necesitan comprensión sino memorización, ya que la forma en que los evalúan al final de una unidad o curso, es haciéndolos recordar personajes, fechas, batallas, y además no consideran que la historia sea de gran complejidad. En este sentido, Ibagón (20I6) reflejan en sus estudios que los estudiantes de bachillerato asumen que la asignatura de historia es una materia fácil, que no presenta dificultad para su aprendizaje, por lo que le dan prioridad a las asignaturas que ellos consideran más difíciles como las ciencias exactas. Otros expresan que el maestro se adueña del turno de clases y lo ocupa con un discurso en el que no existe el intercambio de cada parte, por lo que hace que la asignatura se vuelve más tediosa. Por otra parte, refieren que desde la enseñanza primaria los temas de la asignatura de historia son los mismos, solo un poco más profundos en cada nivel educativo.

Las metodologías y los procedimientos planeados con efectividad y bien utilizados en las clases de historia definen el aprendizaje, pues estas deben ir más allá de las orientaciones del profesor, de las tareas o de las actividades del libro de texto. Se debe dar más participación al estudiante para que sean capaces de pasar de la memorización de contenidos al desarrollo de diálogos y discusiones entre estudiantes y profesor, con el fin de elaborar narrativas -dependiendo de la temática tratada- afines a interpretaciones de situaciones del pasado, que le sirvan como base para comprender el presente. El docente encargado 
del proceso debe lograr que este sea más dinámico, donde el estudiante exponga sus criterios y sus diferentes puntos de vista; tomar más protagonismo en las clases, sintiendo la historia como parte de su vida diaria y no solamente limitarse al abordaje de temas de tiempos ya pasados.

\section{Memoria, cultura y conciencia histórica como parte del proceso de enseñanza/aprendizaje}

La enseñanza de la historia promovió la formación de la identidad nacional, basándose en la transmisión de una narración del pasado. Este tipo de relato se instaló en los discursos pedagógicos y en libros de texto durante gran parte del siglo XX (González, 2006). Es por ello que los libros de texto son el soporte más utilizado para la transmisión de los contenidos y estos ejercen una enorme influencia sobre docentes y alumnos (Monfort, Pagès y Santisteban, 20II). Investigaciones demuestran que aún existen obstáculos teóricos y prácticos alrededor de la enseñanza de la historia, manifiestos en la falta de articulación entre el saber disciplinar y el pedagógico, situación que se refleja en la planeación de los cursos, en la implementación de estrategias de trabajo y en el desarrollo de la clase como tal.

Por estas razones, a la hora de trabajar sobre la base de la formación de un pensamiento dirigido a la acción y a la transformación de la realidad, se debe exigir que el estudiante se sitúe en un aprendizaje significativo; lo cual supone que el conocimiento que se imparte en el currículo y se enseña en la práctica, se plantee de otra manera, donde el estudiante pueda problematizar el conocimiento, construirlo y aprenderlo, utilizando toda clase de fuentes y documentos. Este conocimiento

144 histórico permite ampliar la conciencia personal y social; así como ofrecer al estudiante las herramientas para comprender con mayor claridad la realidad que vive, adaptarse a ella y hasta transformarla (Posada, 20I0).

La principal dificultad en la enseñanza y el aprendizaje del pasado es contextualizar los hechos ocurridos; ante este problema, se requiere 
una comprensión de cómo se llega al conocimiento del pasado, para así emitir una explicación sobre el por qué ocurrieron los hechos de una determinada forma. La historia se interesa más por el significado de los hechos que por los hechos en sí mismos. Es por esto que los docentes deben seleccionar estrategias que ayuden a desarrollar en los estudiantes una mejor comprensión.

En el sentido de la comprensión y la actuación, resulta relevante el trabajo con fuentes y que los estudiantes aprendan a pensar como historiadores (Wineburg, 200I). Las fuentes históricas primarias constituyen la materia prima por excelencia para el conocimiento y comprensión de la historia. Uno de los objetivos fundamentales en la enseñanza de la historia es la formación del pensamiento histórico, este les concede a los alumnos habilidades que les permiten abordar el estudio de la historia y construir su propia representación del pasado. Entonces, pensar históricamente demanda tener conciencia de la temporalidad, capacidad para la representación histórica, imaginación histórica e interpretación de las fuentes históricas (Santisteban, González y Pagès, 20I0).

Un estudio realizado por Llonch (2017) presenta diversas formas de creación de estrategias metodológicas que contribuyen a la generación de ambientes de enseñanza-aprendizaje mediados por las TIC, mejorando así el interés de los estudiantes por determinados contenidos o asignaturas. Este uso de las herramientas tecnológicas en educación debe tener un enfoque que permita la apropiación de elementos con fines educativos, pedagógicos y didácticos, posibilitando también la exploración por parte de los estudiantes. Con este fin, se puede contribuir al aprendizaje del educando en el área de la historia, para evitar caer en métodos de enseñanza reproduccionistas.

En estas formalidades, la UNESCO (2008) establece que los docentes deben desarrollar determinadas competencias tecnológicas que influyan en el proceso de enseñanza-aprendizaje, dentro de las que se encuentran: a) Guiar la práctica docente en relación al uso de las TIC en el aula, b) Enfoque de nociones básicas de TIC, c) Enfoque de profundización del conocimiento, d) Enfoque de generación de 
Conocimiento, entre otras. En la asignatura de historia, estas competencias posibilitan que los alumnos adquirieran tanto los conocimientos de tipo lógico, como el desarrollo de habilidades en el uso de las TIC.

Didácticamente hablando, la historia, en el proceso de enseñarla y hasta de aprenderla, tiene que servir para aproximarse creativamente al pasado, vincularlo con el presente y entender su historicidad. Los estudiosos del tema sugieren que las explicaciones e interpretaciones históricas la hacen más interesante y argumentan que comprender los procesos históricos y ganar conocimientos de base, para entender asuntos actuales, son las razones principales para estudiarla.

El conocimiento de la historia permite tomar conciencia de la situación social del presente, a través del análisis crítico del pasado, convirtiéndose en un instrumento para transformar la realidad. Contribuye a capacitar al alumnado como ciudadano crítico con su tiempo y capaz de comprometerse con su sociedad y actuar para mejorarla. El estudio y el conocimiento histórico son formas para dar sentido a una acción política enraizada en el pasado y dirigida hacia el presente y el futuro, por lo que la historia también se manifiesta como una forma de conocer la cultura del pasado, la cultura popular y la alta cultura. De ahí la importancia del desarrollo de la memoria histórica, tanto para la sociedad, como para el sujeto que en ella se desarrolla. Como categoría cultural, la memoria histórica va ligada a las tradiciones, políticas, valores y costumbres que posibilita incentivar en la persona esas características que le ayudan a construir su identidad, para identificarse con sus raíces y al mismo tiempo marcar diferencias entre otras culturas.

El estudio de la historia se nutre tanto de la memoria como de la educación histórica. Cataño (20II), expone que la memoria histórica es la manera en que los seres humanos tratan de convivir con el pasado para darle significado al presente. Esta le facilita al sujeto la comprensión de los sucesos que se dan en la vida cotidiana y que a su vez tienen perspectivas hacia el futuro; es el esfuerzo consciente de los grupos humanos por encontrar su pasado, sea este real o imaginado. 
Por lo tanto, es uno de los mecanismos del aprendizaje que trae al presente la experiencia de la vida humana. Esto es paralelo con el proceso de aprendizaje significativo, donde el individuo asume la información que tiene relación significativa con la estructura cognitiva emocional y espiritual.

Coudannes (2014) y González (2006), basados en la teoría del filósofo alemán Jörn Rüsen (uno de los principales desarrolladores de esta categoría), expresan que la conciencia histórica es uno de los contrafuertes fundamentales en los individuos para su posicionamiento en la sociedad, pues no es más que una estructura histórica que se apoya en el pasado para darle sentido al presente, en la vida práctica del sujeto, pero también tendrá proyecciones hacia el futuro. El pasado es la plataforma que guiará al individuo a percibir el presente, creando de esta manera una amplia relación entre ellos. Aquí radica la diferencia entre la memoria histórica y conciencia histórica; resumiendo, la memoria está atada al pasado y la conciencia histórica tiene proyección hacia el futuro. Todas las personas son poseedores de una conciencia histórica, que también está muy ligada a la identidad y la cultura histórica, pues es la manera en que se manifiestan y piensan cada uno de los seres humanos.

Tanto la cultura como la memoria histórica marchan al unísono, encontrándose con la conciencia histórica y en su conjunto brindan al sujeto la posibilidad de orientación en la sociedad, para que de igual manera se encuentre él mismo en ella. Es así que la cultura histórica abarca un gran campo de actividades culturales, es vista como un conjunto de fenómenos que con el paso del tiempo se van dando en la sociedad e involucran a todos los que pertenecen a ella. Es un sistema de comunicación en el que se va trasformando la conciencia histórica del hombre; así como sus maneras de sentir, pensar y actuar en sociedad.

Al desarrollar la cultura, la conciencia y la memoria histórica, el hombre alcanza una comprensión más completa de todos los espacios que lo rodean en la sociedad. La cultura es la forma y es también la manera en que emplea su pasado, para relacionarse con el presente 
(Costa, 2009). Es por ello que en el proceso de enseñanza-aprendizaje, estas tres categorías resultan de relevante significación.

En sentido general, el estudio de la historia permite valorar y defender el derecho a las diferencias, lo que presume tolerar y respetar otras ideas, otras costumbres y otras libertades. Sin embargo, una parte importante del profesorado continúa realizando una enseñanza de la historia basada principalmente en el libro de texto, otorgándole poco protagonismo al alumnado (Santiago, 2016). Es por todas estas razones que se hace necesario mejorar la didáctica en el proceso de enseñanza aprendizaje de la historia, de manera que posibilite la construcción de una conciencia histórica en los estudiantes.

\section{Conclusiones}

El papel del educador en el proceso de enseñanza de la asignatura de historia resulta ser de vital importancia para el aprendizaje de los educandos, sin embargo, en las aulas todavía se puede apreciar que los estudiantes no cuentan con las habilidades que exige el programa de esta asignatura, al no contar con los aprendizajes previos, ni el encadenamiento del uso del pasado en el presente y la proyección del futuro.

El trabajo desarrollado por los profesores y estudiantes en las aulas, continúa siendo memorístico, el peso de lo conceptual aún está presente en descrédito de pensar históricamente y darle importancia al pasado en el presente.

Es común que el estudiante pierda el interés en los cursos de historia,

148 precisamente porque no le encuentra sentido al pasado y no hay relación con este presente. Mucho menos pensar que tendrá que ver con el futuro.

La utilización de distintas tecnologías que apoyen y motiven para el logro del interés de los estudiantes por el aprendizaje de los contenidos 
históricos, son necesarias para romper con las didácticas monótonas, que conducen a la simple memorización de los contenidos históricos.

La propuesta didáctica y pedagógica que se plantea en el cuerpo del presente trabajo, es realizar las actividades áulicas relacionadas con la enseñanza y el aprendizaje de la historia, a partir de las fuentes históricas, de preferencia primarias, por lo que se tiene como escenario futuro, la propuesta de una didáctica especial para el logro significativo de los constructos históricos, donde se consideren algunos de los siguientes elementos:

a) Invertir el memorismo de la disciplina histórica por el pensamiento cargado de historicidad, que otorgue significado al pasado en el presente, y la búsqueda futura para la construcción de una sociedad basada en consensos políticos, económicos y sociales.

b) Utilizar la narrativa en las aulas o espacios educativos por parte de profesores y de estudiantes, porque a través del lenguaje se pueden representar los acontecimientos y sus significados simbólicos de lo que han llegado a representar.

c) Considerar la memoria como el guardián de los acontecimientos que afectan al individuo y que estos se transformen en la cultura, necesaria como forma para lograr conformar la conciencia histórica, que le permita a los estudiantes y profesores obtener las pautas de sentir, pensar y actuar en sociedad.

\section{Referencias}

Aceituno, D., y Collao, D. (2018). La historia reciente de Chile: Reflexiones sobre su enseñanza y aprendizaje en tiempos de convergencia cultural. Cadernos de pesquisa: pensamento educaciona $L$, curitiba, I3(33), 52-68.

Benítez, C., Gonda, D., y Pereira, C. (2018). La historia desde la perspectiva de los alumnos del Bachillerato Diversificado. Cuadernos de Investigación Educativa, I(2), I6-19. 
Bertram, C., Wagner, W., \& Trautwein, U. (2017). Learning historical thinking with oral history interviews: A cluster randomized controlled intervention study of oral history interviews in history lessons. American Educational Research Journal, 54(3), 444484 .

Burenheide, B. (2007). I can do this: Revelations on teaching with historical thinking. The History Teacher, 4I(I), 55-6I.

Carretero y López (2009). Estudios cognitivos sobre el conocimiento histórico: aportaciones para la enseñanza y alfabetización histórica. Enseñanza de las ciencias sociales: revista de investigación, 75-89.

Castellanos, M., Estupiñan, T., y Cuesta, M. (2017). Impacto de una herramienta multimedial en el proceso de enseñanzaaprendizaje de la historia en el aula. Revista de Medios y Educación, (50).

Cataño, C. (20II). Jörn Rüsen y la conciencia histórica. Historia y sociedad, (2I), 223-245.

Costa, F. S. (2009). La cultura histórica. Una aproximación diferente a la memoria colectiva. Pasado y Memoria. Revista de Historia Contemporánea, (8), 267-286.

Coudannes, M. (2014). Estudios educativos sobre la conciencia histórica: temas y problemas. Revista Internacional de Ciencias Humanas, $3(2)$.

Cuesta, R. (1998). Clío en las aulas. La enseñanza de la Historia de España entre reformas, ilusiones y rutinas. Madrid: Akal.

Duarte, O. (20I8). La enseñanza de la Historia: innovación y continuidad desde Rafael Altamira/Teaching History: innovation and continuity since Rafael Altamira. Revista Española de Pedagogía, I4I-I55

150 Fronza, M. (2016). La constitución de una didáctica de la historia y la formación docente: por un concepto del aprendizaje histórico. Andamio| Revista de la didáctica de la historia, 2(4), 69-83.

Fuster, C. (20I5). Los estánderes de aprendizaje de la Lomce: ¿Mejorarán la enseñanza y el aprendizaje de la Historia? Revista de Didácticas Específicas, (I2), 27-48. 
Gómez C., Miralles P. y Molina S. (2015). Presentación Evaluación, competencias históricas y educación ciudadana. Revista de estudios sociales, (52), 9-I4.

Gómez C., y Miralles P. (2015). ¿Pensar históricamente o memorizar el pasado? La evaluación de los contenidos históricos en la educación obligatoria en España. Revista de Estudios Sociales, (52), 52-68.

Gómez, C., Rodríguez, R., y Mirete, A. (2016). Relación entre metodología docente y uso de recursos de innovación en la enseñanza de la historia. Un análisis a través de los recuerdos de los maestros en formación. Clío: History and History Teaching. (42).

Gómez, Ortuño y Molina (2014). Aprender a pensar históricamente. Retos para la historia en el siglo XXI. Revista Tempo e Argumento, 6(II), 5-27.

González, M. P. (2006). Conciencia histórica y enseñanza de la historia: una mirada desde los libros de texto. Enseñanza de las ciencias sociales, (5), 2I-30.

Ibagón, N. J. (20I6). Enseñar y aprender historia a partir del análisis de fuentes históricas. Una experiencia formativa en Educación Superior. Voces y silencios. Revista Latinoamericana de educación, 7(I), I2I-I33.

Lamoneda, M. (1998). ¿Cómo enseñamos historia en el nivel medio superior? Revista Mexicana de Investigación Educativa, 3(5), IOI-II2.

Lozano, E. (2013). Patrimonio, Arte y Didáctica de las Ciencias Sociales. Análisis y reflexiones sobre una estrategia de aprendizaje en el marco de la innovación docente. Clío, History and History Teaching, (39), I-I8.

Llonch, N. (20I7). Propuesta de modelo de enseñanza-aprendizaje de la historia en formación de maestros/as a través de la didáctica del objeto. REDU. Revista de Docencia Universitaria, 20I7, vol. 15, vol. I, p. I47-I74.

Marolla, J. y Pagès, J. (2018). Los retos desde la didáctica de las ciencias sociales para trabajar e incluir a las mujeres y su historia en la 
enseñanza: perspectivas de los y las estudiantes chilenos. Revista História Hoje, 7(I3), 253-27I.

Matozzi, I. (1999). La transposición del texto historiográfico: un problema crucial de la didáctica de la historia. Teoría y Didáctica de las Ciencias Sociales, (4), 27-56.

Monfort, N., Pagès, J. y Santisteban, A. (20II). ¿Cómo evaluar el pensamiento histórico del alumnado. En La evaluación en el proceso de enseñanza y aprendizaje de las ciencias sociales. Murcia, España: Asociación Universitaria de Profesorado de Didáctica de las Ciencias Sociales

Montanares, E., y Llancavil, D. (2016). Uso de fuentes históricas en la formación inicial de profesores. Revista Internacional de Investigación en Educación, 8(I7), 85-98.

Ortega, D. (2015). La enseñanza de las Ciencias Sociales, las TIC y el tratamiento de la información y competencia digital (TICD) en el grado de Maestro/a de Educación Primaria de las universidades de Castilla y León. Enseñanza de las Ciencias Sociales, (I4), I2I-I34.

Pérez, L. (20I6). Aprender y enseñar historia a través de la imagen artística: una propuesta didáctica sobre la Revolución Francesa [trabajo de in de máster] de profesor/a en educación secundaria obligatoria, bachillerato, formación profesional y enseñanza de idiomas CURSO 2015 - 2016.

Posada, R. (20I0). La enseñanza de la historia: perspectivas y retos en la educación superior. Estudio de caso enseñanza de la historia en la licenciatura en ciencias Sociales de la universidad de caldas. Revista Latinoamericana de Estudios Educativos, 6(2), I79-203.

Prats, J. (20I7). Retos y dificultades para la enseñanza de la historia. En La historia en el aula: innovación docente y enseñanza de la historia en la educación secundaria (pp. 15-32). Editorial Milenio.

Prats, J. y Santacana, J. (1998). Enseñar historia y geografía. Principios básicos.

Recuperado de: http://www.ub.edu/histodidactica/index.php?option=com cont ent\&view=article\&id=75:ensenar-historia-y-geografiaprincipios-basicos\&catid=24: articulos-cientificos\&Itemid $=I I 8$. 
Prats, J., y Santacana, J. (20II). Enseñar a pensar históricamente: la clase como simulación de la investigación histórica. En Didáctica de la Geografía y la Historia (pp. 67-87). Secretaría General Técnica.

Ramallo, F. y Porta, L. (20I7). (Re) Fundar un relato desde la memoria escolar: narrativas y prácticas en la historia del bachillerato argentino. Journal for Educators, Teachers and Trainers, 8(I), 35-46.

Sáiz, J., y Gómez, C. J. (20I6). Investigar el pensamiento histórico y narrativo en la formación del profesorado: fundamentos teóricos y metodológicos. Revista Electrónica Interuniversitaria de Formación del Profesorado, I9(I), I75-190.

Sáiz, J., y Fuster, C. (20I4). Memorizar historia sin aprender pensamiento histórico: las PAU de Historia de España. Revista Investigación en la Escuela, (84), 47-57.

Sáiz, J., y López, R. (20I5). Competencias y narrativas históricas: el pensamiento histórico de estudiantes y futuros profesores españoles de educación secundaria. Revista de Estudios Sociales, (52), 87-IOI.

Santiago, J. (2016). La acción didáctica de las Ciencias Sociales y el desarrollo del pensamiento crítico. Educación $y$ Humanismo, 18(31), 241-256.

Santisteban, A., González, N., y Pagès, J. (20I0). Una investigación sobre la formación del pensamiento histórico. Metodología de investigación en Didáctica de las Ciencias Sociales, II5-I28.

Sanz, P., Molero, J.M., y Rodríguez, D. (20I7). La historia en el aula. Innovación docente y enseñanza de la historia en la educación secundaria. Historia y Memoria de la Educación, (6), 553-555.

Schmidt, M. A. (20I6). Globalización y la política de formación del profesor de historia en Brasil. Perspectiva Educacional, Formación de Profesores, 55(I), 38-50.

Schmidt, M. A. (20I7). ¿Qué hacen los historiadores cuando enseñan la Historia? Contribuciones de la teoría de Jörn Rüsen para el aprendizaje y el método de enseñanza de la Historia. Clio $\mathcal{E}$ asociados. La historia enseñada, (24), 26-37

Stearns, P. Seixas, P. Y Wineburg, S. (ed.). 2000. Knowing, Teaching and Learning History. National and International Perspectives. 
Nueva York - Londres: New York University Press. United States of America.

Tellez, I. (2020). El tratamiento de historia local en los escolares de la educación primaria. Revista Electrónica de Psicología Iztacala, 23(3), 87I-894.

VanSledright, A. (20I4), Assessing Historical Thinking and Understanding. Innovation design for new standards. Nueva York: Routledge.

Wineburg, S (200I). Historical thinking and other unnatural acts. Charting the future of teaching the past. Chicago: Temple University Press.

Zhao, Y., y Hoge, J. D. (2005). Lo que los estudiantes y maestros de primaria dicen acerca de los estudios sociales. Los estudios sociales, 96(5), 2I6-22I.

Este artículo se publica bajo una licencia de Creative Commons Reconocimiento-NoComercial 4.0

Internacional, y puede ser usados gratuitamente para fines no comerciales, dando los créditos a los autores y a la revista. 